\title{
FACTORIZATION IN CODIMENSION TWO IDEALS OF GROUP ALGEBRAS
}

\author{
G. A. WILLIS
}

\begin{abstract}
Let $G$ be a finitely generated group and $I$ be a closed, two-sided ideal with codimension two in $L^{\prime}(G)$. Then the linear span of the set of all products in $I$ is equal to $I$.
\end{abstract}

Let $A$ be a complex algebra. For $I, J \subseteq A$, define $I J=\left\{\sum_{k=1}^{n} a_{k} b_{k} \mid n \in \mathbf{N}\right.$; $\left.a_{k} \in I, b_{k} \in J, k=1, \ldots, n\right\}$, and abbreviate $I I$ as $I^{2}$. It is clear that if $I$ is an ideal in $A$, then $I^{2} \subseteq I$ (by ideal will always be meant a two-sided ideal). The ideal $I$ is said to be idempotent if $I^{2}=I$.

Now suppose that $A$ is also a Banach algebra. Then a question which arises in connection with automatic continuity problems for $A$ is whether $I$ is idempotent whenever $I$ is a closed finite-codimensional ideal in $A$. For example, see $[2, \S 6]$ and $[3]$.

This question is particularly interesting when $A$ is the group algebra, $L^{1}(G)$, of a locally compact group $G$, as there are several classes of groups such that every finite-codimensional ideal in $L^{1}(G)$ is idempotent. For example, it is an immediate consequence of Theorem 2 in [5] that, if $G$ is amenable, then every closed, finite-codimensional ideal in $L^{1}(G)$ has a bounded approximate identity. Hence, by Cohen's factorization theorem [1, Theorem 11.10], every such ideal is idempotent. It is also shown in [8] that, if $G$ is connected, then every closed, finite-codimensional ideal in $L^{1}(G)$ is idempotent. Furthermore, in [6] it is shown that closed ideals with codimension one in $L^{1}(G)$ are idempotent for every $G$. Every group algebra has at least one codimension one ideal, namely the augmentation ideal $I_{0}(G)=$ $\left\{f \in L^{1}(G) \mid \int_{G} f d x=0\right\}$.

This paper is concerned with ideals with codimension two in $L^{1}(G)$. The main theorem deals with finitely generated groups. Since a finitely generated group $G$ is countable, Haar measure on $G$ will be discrete. Hence we may normalize it to be counting measure and take $L^{1}(G)$ to be the set of functions $f$ on $G$ with

$$
\|f\|=\sum_{x \in G}|f(x)|<\infty
$$

The function which takes the value one at $x$ and is zero elsewhere will be denoted by $\bar{x}$. If 1 is the identity element of $G$, then $\bar{l}$ is a multiplicative identity for $L^{1}(G)$.

Received by the editors September 9, 1982.

1980 Mathematics Subject Classification. Primary 43A20; Secondary 46H10. 
THEOREM 1. Let $G$ be a finitely generated group and I be a closed, two-sided ideal with codimension two in $L^{1}(G)$. Then I is idempotent.

Proof. Consider $L^{1}(G) / I$. It is a two-dimensional complex algebra with unit, and is semisimple by [3, Lemma 3.1]. (Alternatively, $L^{1}(G) / I$ may be shown to be semisimple in the following way. Let $\rho: L^{1}(G) \rightarrow L^{1}(G) / I$ be the quotient homomorphism and $R$ be the radical of $L^{1}(G) / I$. Then if $R \neq(0), \rho^{-1}(R)$ is a nonidempotent codimension one ideal in $L^{1}(G)$, which contradicts the theorem of [6].) Hence $L^{1}(G) / I$ is isomorphic to $\mathbf{C} \oplus \mathbf{C}$ and so there are multiplicative linear functionals $\phi_{0}$ and $\phi_{1}$ on $L^{1}(G)$ such that $I=\operatorname{ker} \phi_{0} \cap \operatorname{ker} \phi_{1}$.

Let $\chi_{0}$ and $\chi_{1}$ be the characters on $G$ such that

$$
\phi_{j}(f)=\sum_{x \in G} f(x) \chi_{j}(x) \quad\left(f \in L^{1}(G), j=0,1\right)
$$

(see [4, Corollary 23.7]). Define an automorphism $T$ of $L^{\prime}(G)$ by

$$
(T f)(x)=f(x) \chi_{0}(x) \quad\left(x \in G, f \in L^{1}(G)\right) .
$$

Then $T(I)$ is a codimension two ideal in $L^{\prime}(G)$ and in order to show that $I$ is idempotent it will suffice to show that $T(I)$ is idempotent. Hence, replacing $I$ by $T(I)$ and $\phi_{j}$ by $\phi_{j} \circ T^{-1}$, we may suppose that $\chi_{0}$ is the trivial character and ker $\phi_{0}$ is the augmentation ideal of $L^{1}(G)$.

Now suppose that $G$ is generated by $n$ elements $y_{1}, y_{2}, \ldots, y_{n}$ and let $\mathbf{F}_{n}$ be the free group on $n$ generators $x_{1}, x_{2}, \ldots, x_{n}$. Then there is a surjective group homomorphism $q: \mathbf{F}_{n} \rightarrow G$ defined by

$$
q\left(x_{i}\right)=y_{i}, \quad i=1,2, \ldots, n,
$$

and a surjective algebra homomorphism $Q: L^{1}\left(\mathbf{F}_{n}\right) \rightarrow L^{1}(G)$ defined by

$$
(Q f)(y)=\sum_{x \in q^{-1}(y)} f(x) \quad\left(y \in G, f \in L^{\prime}\left(\mathbf{F}_{n}\right)\right) .
$$

It is clear that $Q^{-1}(I)$ is a codimension two ideal in $L^{\prime}\left(\mathbf{F}_{n}\right)$ and that $I$ is idempotent if $Q^{-1}(I)$ is. Hence it will suffice to prove the theorem in the case when $G=\mathbf{F}_{n}$.

If $\chi_{1}$ was the trivial character, then we would have that $\phi_{0}=\phi_{1}$ and $I$ would have codimension one. Thus $\chi_{1}$ is not trivial and we may suppose that $\chi_{1}\left(x_{1}\right) \neq 1$. Now if $\chi_{1}\left(x_{i}\right)=1$ for some $i$, then $\chi_{1}\left(x_{1} x_{i}\right) \neq 1$ and $\left\{x_{1}, \ldots, x_{i-1}, x_{1} x_{i}, x_{i+1}, \ldots, x_{n}\right\}$ still generates $\mathbf{F}_{n}$ freely. Thus, by replacing $x_{i}$ with $x_{1} x_{i}$ if necessary, we may further suppose that $\chi_{1}\left(x_{i}\right) \neq 1$ for each $i$.

It is convenient to introduce a little more notation. Let $2^{n}$ denote the set of all functions on $\{1,2, \ldots, n\}$ taking values 0 or 1 . For each $t$ in $2^{n}$, define $e(t)$ in $L^{1}\left(\mathbf{F}_{n}\right)$ by $e(t)=\left(c_{1} \overline{1}-\bar{x}_{1}\right) *\left(c_{2} \overline{1}-\bar{x}_{2}\right) * \cdots *\left(c_{n} \overline{1}-\bar{x}_{n}\right)$, where $c_{i}=1$ if $t(i)=0$ and $c_{i}=\chi_{1}\left(x_{i}\right)$ if $t(i)=1$. The constant functions in $2^{n}$ with values 0 and 1 will be denoted by 0 and $l$ respectively. We will require the following lemma.

Lemma. Let $t$ be in $2^{n} \backslash\{0,1\}$. Then $e(t)$ is in $I^{2}$.

Proof. Since $t$ is not constant, there is an $i$ between 1 and $n-1$ such that either

$$
e(t)=\cdots *\left(\overline{1}-\bar{x}_{i}\right) *\left(\chi_{1}\left(x_{i+1}\right) \overline{1}-\bar{x}_{i+1}\right) * \cdots
$$


or

$$
e(t)=\cdots *\left(\chi_{1}\left(x_{i}\right) \overline{1}-\bar{x}_{i}\right) *\left(\overline{1}-\bar{x}_{i+1}\right) * \cdots .
$$

Thus, since $I^{2}$ is an ideal in $L^{1}\left(\mathbf{F}_{n}\right)$, it will suffice to show that

$$
\left(\overline{1}-\bar{x}_{i}\right) *\left(\chi_{1}\left(x_{i+1}\right) \overline{1}-\bar{x}_{i+1}\right) \text { and }\left(\chi_{1}\left(x_{i}\right) \overline{1}-\bar{x}_{i}\right) *\left(\overline{1}-\bar{x}_{i+1}\right)
$$

are in $I^{2}$ for each $i$ between 1 and $n-1$.

For this, choose $h$ in $L^{1}\left(\mathbf{F}_{n}\right)$ such that $h$ is in $\operatorname{ker} \phi_{1}$ and $\overline{1}-h$ is in $\operatorname{ker} \phi_{0}$. Then

$$
\begin{aligned}
& \left(\overline{1}-\bar{x}_{i}\right) *\left(\chi_{1}\left(x_{i+1}\right) \overline{1}-\bar{x}_{i+1}\right)=h *\left(\overline{1}-\bar{x}_{i}\right) *\left(\chi_{1}\left(x_{i+1}\right) \overline{1}-\bar{x}_{i+1}\right) \\
& \quad+(\overline{1}-h) *\left(\chi_{1}\left(x_{i+1}\right) \overline{1}-\bar{x}_{i+1}\right) *\left(\overline{1}-\bar{x}_{i}\right)+(\overline{1}-h) *\left(\overline{x_{i} x_{i+1}}-\overline{x_{i+1} x_{i}}\right) .
\end{aligned}
$$

Now $\overline{1}-\bar{x}_{i}$ has a square root defined by the binomial expansion

$$
\left(\overline{1}-\bar{x}_{i}\right)^{1 / 2}=\sum_{k=0}^{\infty}\left(\begin{array}{c}
1 / 2 \\
k
\end{array}\right)\left(\bar{x}_{i}\right)^{* k},
$$

where the series converges because $\left(\begin{array}{c}1 / 2 \\ k\end{array}\right)$ is an $l^{1}$-sequence and because $\left\|\left(\bar{x}_{i}\right)^{* k}\right\|=1$ for each $k$. The square root is in $\operatorname{ker} \phi_{0}$ because $0=\phi_{0}\left(\overline{1}-\bar{x}_{i}\right)=\phi_{0}\left(\left(\overline{1}-\bar{x}_{i}\right)^{1 / 2}\right)^{2}$. Hence

$$
\begin{aligned}
h *\left(\overline{1}-\bar{x}_{i}\right) & *\left(\chi_{1}\left(x_{i+1}\right) \overline{1}-\bar{x}_{i+1}\right) \\
& =\left[h *\left(\overline{1}-\bar{x}_{i}\right)^{1 / 2}\right] *\left[\left(\overline{1}-\bar{x}_{i}\right)^{1 / 2} *\left(\chi_{1}\left(x_{i+1}\right) \overline{1}-\bar{x}_{i+1}\right)\right] \\
& \in\left[\operatorname{ker} \phi_{1} * \operatorname{ker} \phi_{0}\right] *\left[\operatorname{ker} \phi_{0} * \operatorname{ker} \phi_{1}\right] \subseteq I^{2}
\end{aligned}
$$

It may be shown in the same way that $(\overline{1}-h) *\left(\chi_{1}\left(x_{i+1}\right) \overline{1}-\bar{x}_{i+1}\right) *\left(\overline{1}-\bar{x}_{i}\right)$ is in $I^{2}$. Finally,

$$
\overline{x_{i} x_{i+1}}-\overline{x_{i+1} x_{i}}=\left(\overline{1}-\left(x_{i+1} x_{i} x_{i+1}^{-1} x_{i}^{-1}\right)^{-}\right) *\left(\overline{x_{i} x_{i+1}}\right),
$$

where $\overline{1}-\left(x_{i+1} x_{i} x_{i+1}^{-1} x_{i}^{-1}\right)^{-}$is in $I$ and has a square root which must also be in $I$ because $I=\operatorname{ker} \phi_{0} \cap \operatorname{ker} \phi_{1}$. Hence $\overline{x_{i} x_{i+1}}-\overline{x_{i+1} x_{i}}$ is in $I^{2}$ also. It now follows from (1) that $\left(\overline{1}-\bar{x}_{i}\right) *\left(\chi_{1}\left(x_{i+1}\right) \overline{1}-\bar{x}_{i+1}\right)$ is in $I^{2}$ for each $i$. That $\left(\chi_{1}\left(x_{i}\right) \overline{1}-\bar{x}_{i}\right) *(\overline{1}-$ $\left.\bar{x}_{i+1}\right)$ is in $I^{2}$ also may be proved in the same way.

We now continue the proof of the theorem. Every $x$ in $\mathbf{F}_{n}$ may be written uniquely in the form $x=x_{1}^{k_{1}} x_{2}^{k_{2}} \cdots x_{n}^{k_{n}} y$, where $k_{1}, k_{2}, \ldots, k_{n}$ are integers and $y$ is in $\mathbf{F}_{n}^{\prime}$, the commutator subgroup of $\mathbf{F}_{n}$. For each $i$ between 1 and $n$, let $Z_{i}=\left\{x_{i}^{k_{i}} \cdots x_{n}^{k_{n}} y\right\}$ $\left.k_{i}, \ldots, k_{n} \in \mathbf{Z}, y \in \mathbf{F}_{n}^{\prime}\right\}$ and let $\left\langle x_{i}\right\rangle$ be the subgroup of $\mathbf{F}_{n}$ generated by $x_{i}$. Let $Z_{n+1}=\mathbf{F}_{n}^{\prime}$. Then $Z_{1}=\mathbf{F}_{n}$ and $Z_{i+1} \subseteq Z_{i}, i=1,2, \ldots, n$.

Let $i$ be between 1 and $n$ and denote by $L^{1}\left(\left\langle x_{i}\right\rangle\right)$ and $L^{1}\left(Z_{i}\right)$ the spaces of functions in $L^{\prime}\left(\mathbf{F}_{n}\right)$ with support in $\left\langle x_{i}\right\rangle$ and $Z_{i}$ respectively. Since $\chi_{1}\left(x_{i}\right) \neq 1$, $L^{1}\left(\left\langle x_{i}\right\rangle\right) \cap I$ has codimension two in $L^{1}\left(\left\langle x_{i}\right\rangle\right)$. Hence we may define bounded linear functionals $\eta, \xi$ on $L^{1}\left(\left\langle x_{i}\right\rangle\right)$ and a bounded linear operator $T: L^{1}\left(\left\langle x_{i}\right\rangle\right) \rightarrow L^{1}\left(\left\langle x_{i}\right\rangle\right)$ $\cap I$ in a unique way such that for each $f$ in $L^{1}\left(\left\langle x_{i}\right\rangle\right)$,

$$
f=\eta(f)\left(\overline{1}-\bar{x}_{i}\right)+\xi(f)\left(\chi_{1}\left(x_{i}\right) \overline{1}-\bar{x}_{i}\right)+T(f) .
$$


Furthermore, $L^{1}\left(\left\langle x_{i}\right\rangle\right)$ is a subalgebra of $L^{1}\left(\mathbf{F}_{n}\right)$ isomorphic to $L^{1}(\mathbf{Z})$ (the group algebra of the integers) and $L^{1}\left(\left\langle x_{i}\right\rangle\right) \cap I$ is a closed ideal in $L^{1}\left(\left\langle x_{i}\right\rangle\right)$. Hence, by [5, Theorem 2], $L^{1}\left(\left\langle x_{i}\right\rangle\right) \cap I$ has a bounded approximate identity. Let $J_{i}$ be the closed linear span of $\left(L^{\prime}\left(\left\langle x_{i}\right\rangle\right) \cap I\right) * L^{\prime}\left(\mathbf{F}_{n}\right)$. Then it is clear that $J_{i}$ is a left Banach module over $L^{1}\left(\left\langle x_{i}\right\rangle\right) \cap I$, that $J_{i}$ is contained in $I$, and that $L^{1}\left(\left\langle x_{i}\right\rangle\right) \cap I$ has a bounded approximate identity for $J_{i}$ (in the sense of $[1,11.8]$ ).

Now for each $f$ in $L^{1}\left(Z_{i}\right)$ and each $z$ in $Z_{i+1}$, let $f_{z}$ be the function in $L^{1}\left(\left\langle x_{i}\right\rangle\right)$ defined by $f_{z}\left(x_{i}^{k}\right)=f\left(x_{i}^{k} z\right)$. Then $\Sigma_{z \in Z_{I-1}}\left\|f_{z}\right\|=\|f\|$ and $f=\Sigma_{z \in Z_{1+1}} f_{z} * \bar{z}$. Hence, by (2),

$$
\begin{aligned}
f= & \left(\overline{1}-\bar{x}_{i}\right) *\left(\sum_{z \in Z_{i+1}} \eta\left(f_{z}\right) \bar{z}\right)+\left(\chi_{1}\left(x_{i}\right) \overline{1}-\bar{x}_{i}\right) *\left(\sum_{z \in Z_{i+1}} \xi\left(f_{z}\right) \bar{z}\right) \\
& +\sum_{z \in Z_{i+1}} T\left(f_{z}\right) * \bar{z} .
\end{aligned}
$$

Put $h=\sum_{z \in Z_{i+1}} T\left(f_{z}\right) * \bar{z}$. Then $h$ is in $J_{i}$ and so, by Cohen's factorization theorem [ $\mathbf{1}$, 11.10], there are $a$ in $L^{1}\left(\left\langle x_{i}\right\rangle\right) \cap I$ and $h^{\prime}$ in $J_{i}$ such that $h=a * h^{\prime}$. Since both $L^{1}\left(\left\langle x_{i}\right\rangle\right) \cap I$ and $J_{i}$ are contained in $I$, it follows that $h$ is in $I^{2}$. Hence, putting $\Sigma_{z \in Z_{i+1}} \eta\left(f_{z}\right) \bar{z}=g_{0}$ and $\Sigma_{z \in Z_{1+1}} \xi\left(f_{z}\right) \bar{z}=g_{1}$, we have shown that for each $f$ in $L^{1}\left(Z_{i}\right)$,

$$
f=\left(\overline{1}-\bar{x}_{i}\right) * g_{0}+\left(\chi_{1}\left(x_{i}\right) \overline{1}-\bar{x}_{i}\right) * g_{1}+h,
$$

where $g_{0}$ and $g_{1}$ are in $L^{1}\left(Z_{i+1}\right)$ and $h$ is in $I^{2}$.

In particular, if $f$ is in $L^{1}\left(\mathbf{F}_{n}\right)$, then

$$
f=\left(\overline{1}-\bar{x}_{1}\right) * g_{0}+\left(\chi_{1}\left(x_{1}\right) \overline{1}-\bar{x}_{1}\right) * g_{1}+h,
$$

where $g_{0}$ and $g_{1}$ are in $L^{1}\left(Z_{2}\right)$ and $h$ is in $I^{2}$. Next, applying (3) to $g_{0}$ and $g_{1}$ and remembering that $I^{2}$ is an ideal, we find that

$$
\begin{aligned}
f= & \left(\overline{1}-\bar{x}_{1}\right) *\left(\overline{1}-\bar{x}_{2}\right) * g_{00}+\left(\overline{1}-\bar{x}_{1}\right) *\left(\chi_{1}\left(x_{2}\right) \overline{1}-\bar{x}_{2}\right) * g_{01} \\
& +\left(\chi_{1}\left(x_{1}\right) \overline{1}-\bar{x}_{1}\right) *\left(\overline{1}-\bar{x}_{2}\right) * g_{10} \\
& +\left(\chi_{1}\left(x_{1}\right) \overline{1}-\bar{x}_{1}\right) *\left(\chi_{1}\left(x_{2}\right) \overline{1}-\bar{x}_{2}\right) * g_{11}+h^{\prime \prime},
\end{aligned}
$$

where $g_{00}, g_{01}, g_{10}, g_{11}$ are in $L^{1}\left(Z_{3}\right)$ and $h^{\prime \prime}$ is in $I^{2}$. Now applying (3) to $g_{00}, g_{01}, g_{10}$ and $g_{11}$ and so on, we find after $n$ steps that for each $f$ in $L^{1}\left(\mathbf{F}_{n}\right)$,

$$
f=\sum_{t \in 2^{n}} e(t) * g(t)+\psi,
$$

where $g(t)$ is in $L^{1}\left(Z_{n+1}\right)\left(=L^{1}\left(\mathbf{F}_{n}^{\prime}\right)\right)$ for every $t$ in $2^{n}$ and $\psi$ is in $I^{2}$. Finally, by the lemma, $e(t)$ is in $I^{2}$ for each $t$ other than 0 or $l$ and so for every $f$ in $L^{1}\left(\mathbf{F}_{n}\right)$,

$$
f=e(0) * g(0)+e(1) * g(1)+\psi^{\prime},
$$

where $g(0)$ and $g(I)$ are in $L^{1}\left(\mathbf{F}_{n}^{\prime}\right)$ and $\psi^{\prime}$ is in $I^{2}$.

Let $f$ be in $I$. Then,

$$
0=\phi_{1}(f)=\phi_{1}(e(0)) \phi_{1}(g(0))+\phi_{1}(e(l)) \phi_{1}(g(l))+\phi_{1}\left(\psi^{\prime}\right) .
$$

Since $\phi_{1}\left(\psi^{\prime}\right)=0=\phi_{1}(e(1))$ and $\phi_{1}(e(0)) \neq 0$, it follows that $\phi_{1}(g(0))=0$. Thus,

$$
0=\sum_{x \in \mathbf{F}_{n}} g(0)(x) \chi_{1}(x)=\sum_{y \in \mathbf{F}_{n}^{\prime}} g(0)(y) \text {, }
$$


because $g(0)$ is supported in $\mathbf{F}_{n}^{\prime}$ and characters are trivial on $\mathbf{F}_{n}^{\prime}$. Hence $g(0)$ is in $I_{0}\left(\mathbf{F}_{n}^{\prime}\right)$. Similarly, using the fact that $\phi_{0}(f)=0$, it may be shown that $g(l)$ is in $I_{0}\left(\mathbf{F}_{n}^{\prime}\right)$. Now $I_{0}\left(\mathbf{F}_{n}^{\prime}\right)$ is contained in $I$ and is idempotent by [6]. Hence,

$$
g(0), g(l) \in I_{0}\left(\mathbf{F}_{n}^{\prime}\right)=I_{0}\left(\mathbf{F}_{n}^{\prime}\right)^{2} \subseteq I^{2},
$$

and so $f$ is in $I^{2}$. Therefore $I$ is idempotent.

There are still many unanswered questions concerning factorization in finite-codimensional ideals of group algebras. Some of these questions are discussed in more detail in $\$ 4$ of [7]. It is mentioned there, without proof, that the above theorem may be used to show that, if $G$ is finitely generated, then every ideal $I$ such that $L^{1}(G) / I$ is commutative and finite dimensional is idempotent. In particular it follows that, if $G$ is finitely generated, then every ideal with codimension three in $L^{1}(G)$ is idempotent. We now give a proof of this fact.

THEOREM 2. Let $A$ be a complex algebra such that $A^{2}=A$. Suppose that

(i) every ideal with codimension one in $A$ is idempotent; and

(ii) if $I$ and $J$ are ideals with codimension one in $A$, then $I J=J I$.

Then every ideal $I$ in $A$ such that $A / I$ is finite dimensional and commutative is idempotent.

Proof. By adjoining an identity we may assume that $A$ has a unit, which we will denote by 1 . Let $I$ be an ideal in $A$ with $A / I$ commutative and finite dimensional, but with $\operatorname{dim}(A / I) \geqslant 2$. We may suppose that every ideal properly containing $I$ is idempotent.

Denote by $\rho$ the quotient map from $A$ to $A / I$ and let $R$ be the radical of $A / I$. If $R \neq(0)$, then $R^{2} \subsetneq R$ because $R$ is a finite-dimensional radical algebra. Hence, if $R \neq(0),\left(\rho^{-1}(R)\right)^{2} \subseteq \rho^{-1}\left(R^{2}\right) \neq \rho^{-1}(R)$, and so $\rho^{-1}(R)$ is a nonidempotent ideal in $A$ which properly contains $I$. Therefore $R=(0)$, and so $A / I$ is semisimple. It follows that $A / I$ is isomorphic to the direct sum of $n$ copies of $\mathbf{C}$, where $n$ is the codimension of $I$. Hence, there are ideals $I_{1}, I_{2}, \ldots, I_{n}$ with codimension one in $A$ such that $I=\cap_{j=1}^{n} I_{j}$.

Since $I_{1}$ and $I_{2}$ are distinct codimension one ideals in $A$, there is an $h$ in $I_{1}$ such that $1-h$ is in $I_{2}$. Hence, for each $f$ in $I, f=f h+f(1-h)$, and so $I \subseteq I I_{1}+I I_{2}$.

Similarly, if $j_{1}, j_{2}, \ldots, j_{k}$ are distinct integers between 1 and $n$, where $k \leqslant n-2$, and $i_{1}$ and $i_{2}$ are distinct integers not equal to any of the $j_{k}$ 's then

$$
I I_{j_{1}} I_{j_{2}} \cdots I_{j_{h}} \subseteq I I_{j_{1}} \cdots I_{j_{h}} I_{i_{1}}+I I_{j_{1}} \cdots I_{j_{h}} I_{i_{2}}
$$

where now $\left\{j_{1}, j_{2}, \ldots, j_{k}, i_{1}\right\}$ and $\left\{j_{1}, j_{2}, \ldots, j_{k}, i_{2}\right\}$ are sets of distinct integers. We may thus show that $I$ is contained in the sum of ideals of the form $I I_{j_{2}} I_{j_{3}} \cdots I_{j_{n}}$, where $j_{2}, j_{3}, \ldots, j_{n}$ are distinct integers between 1 and $n$. However, $I \subseteq \cap_{j=1}^{n} I_{j}$, and so $I I_{j_{2}} I_{j_{3}} \cdots I_{j_{n}} \subseteq I_{j_{1}} I_{j_{2}} \cdots I_{j_{n}}$, where $j_{1}$ is chosen to be the integer between 1 and $n$ which does not appear in the list $j_{2}, j_{3}, \ldots, j_{n}$. Therefore,

$$
I \subseteq \sum_{\pi \in S_{n}}\left(I_{\pi(1)} I_{\pi(2)} \cdots I_{\pi(n)}\right)
$$

where $S_{n}$ is the group of permutations of $\{1,2, \ldots, n\}$. 
Now,

$$
\begin{aligned}
I_{1} I_{2} \cdots I_{n} & =\left(I_{1}\right)^{2}\left(I_{2}\right)^{2} \cdots\left(I_{n}\right)^{2}, \quad \text { by }(\mathrm{i}) \\
& =I_{1} I_{2} I_{1} I_{2}\left(I_{3}\right)^{2} \cdots\left(I_{n}\right)^{2}, \quad \text { by (ii), } \\
& =\left(I_{1} I_{2} I_{3}\right)^{2}\left(I_{4}\right)^{2} \cdots\left(I_{n}\right)^{2}, \quad \text { by (ii) applied twice, } \\
& =\cdots \\
& =\left(I_{1} I_{2} \cdots I_{n}\right)^{2} \subseteq I^{2}
\end{aligned}
$$

Similarly, $I_{\pi(1)} I_{\pi(2)} \cdots I_{\pi(n)} \subseteq I^{2}$ for every $\pi$ in $S_{n}$. Therefore, by (4), $I \subseteq I^{2}$.

Remarks. (a) The codimension one ideals of $A$ will satisfy (ii) either if $A$ is commutative (clear) or if the codimension two ideals of $A$ are idempotent ( $I J \subseteq I \cap$ $\left.J=(I \cap J)^{2} \subseteq J I\right)$.

(b) If $A$ is a Banach algebra and $A^{2}=A$, then every codimension one ideal in $A$ is closed. Hence we need only verify that (i) and (ii) hold for the closed codimension one ideals of $A$. Furthermore, in the course of the proof it was shown that if $A$ satisfies (i) and (ii) and if $I$ is an ideal in $A$ such that $A / I$ is commutative and finite dimensional, then $I$ is the intersection of codimension one ideals. Hence under these conditions $I$ is closed.

(c) Suppose that $A$ satisfies the conditions of the theorem, then all ideals with codimension two or three in $A$ are idempotent. If $I$ has codimension two, then $A / I$ is semisimple (otherwise the inverse image of $\operatorname{Rad}(A / I)$ will be a nonidempotent codimension one ideal). Hence $A / I$ is isomorphic to $\mathbf{C} \oplus \mathbf{C}$ (the only semisimple, two-dimensional complex algebra). Now $\mathbf{C} \oplus \mathbf{C}$ is commutative and so $I$ is idempotent by the theorem. A similar argument proves the result for codimension three ideals. The result does not necessarily hold for codimension four ideals because there is a four-dimensional noncommutative, semisimple complex algebra-namely the $2 \times 2$ complex matrices.

\section{REFERENCES}

1. F. F. Bonsall and J. Duncan, Complete normed algebras, Springer-Verlag, New York, 1973.

2. H. G. Dales, Automatic continuity: a survey, Bull. London Math. Soc. 10 (1978), 129-183.

3. H. G. Dales and G. A. Willis, Cofinite ideals in Banach algebras, and finite-dimensional representations of group algebras. (Proc. Conf. Automatic Continuity...), Lecture Notes in Math., vol. 975, SpringerVerlag, New York, 1982.

4. E. Hewitt and K. A. Ross, Abstract harmonic analysis. I, Springer-Verlag, Berlin, 1963.

5. T.-S. Liu, A. van Rooij and J.-K. Wang, Projections and approximate identities for ideals in group algebras, Trans. Amer. Math. Soc. 166 (1972), 401-410.

6. G. Willis, Factorization in codimension one ideals of group algebras, Proc. Amer. Math. Soc. 86 (1982), 599-601.

7. The continuity of derivations from group algebras and factorization in cofinite ideals (Proc. Conf. Automatic Continuity... ), Lecture Notes in Math., vol. 975, Springer-Verlag, New York, 1982.

8. The continuity of derivations from group algebras: factorizable and connected groups (to appear).

School of Mathematics, University of New South Wales, Kensington, N.S.W., 2033, Australia

Current address: Department of Mathematics, Dalhousie University, Halifax, N.S. B3H 4H6, Canada 